\title{
Análisis de registros de comportamientos previos para la toma de decisiones. Aplicación para la dirección de proyectos software
}

\author{
Analysis of records of previous behaviour for decision-making. \\ Application for software project management
}

Arturo Peralta Martín-Palomino ${ }^{1}$

Recibido 23 de diciembre de 2015, aceptado 9 de marzo de 2017

Received: December 23, 2015 Accepted: March 9, 2017

\begin{abstract}
RESUMEN
El PSP (Proceso de Software Personal) es un conjunto de prácticas dirigidas a la gestión del tiempo y a la mejora de la productividad personal aplicadas sobre todo dentro de tareas de Ingeniería del Software. Estas técnicas pueden ser aprovechadas con el fin de mejorar la gestión de las tareas tanto de directivos para la toma de decisiones como empleados de empresas de desarrollo de software. En este trabajo se presentan diferentes experiencias en la aplicación de las prácticas más básicas de este proceso, mostrando como resultados su impacto tanto en la planificación de las agenda de tareas de directivos como en el desarrollo de proyectos software por un equipo de desarrollo.
\end{abstract}

Palabras clave: Proceso de software personal, planificación de proyectos, estimación de tareas, gestión de tiempos, reconocimiento de patrones de comportamiento.

\begin{abstract}
The PSP (Personal Software Process) is a set of practices aimed at time management and personal productivity improvement, applied primarily within software engineering tasks. These techniques can be exploited in order to improve the management of tasks both managers for decision-making and employees of software development companies. In this paper is presented different experiences during the application of the most basic practices of this process, presenting its impact results in the planning of the agenda management tasks such as software development projects for the development team.
\end{abstract}

Keywords: Personal software process, project planning, estimation tasks, time management, behavioural pattern recognition.

\section{INTRODUCCIÓN}

En la actualidad, uno de los principales problemas habituales al que se enfrentan tanto directivos como empleados es la construcción de una agenda semanal eficiente. En ella, además de las tareas que podrían ser consideradas como básicas para la semana, deberían reflejarse además aquellas relativas a tareas de apoyo o de gestión, como podrían ser la preparación de reuniones, elaboración de informes, presentaciones o auto-estudio.

Si consideramos el problema de la gestión del tiempo en su esencia como una causa de estrés, ampliamente

1 Departamento de Sistemas de Información. EAE Business School. Joaquín Costa 41. Madrid, España. E-mail: aperalta@campus.eae.es 
estudiada dentro de campos como la psicología, podremos determinar que ciertas disciplinas como la terapia cognitiva nos van a ofrecer herramientas útiles para resolver este problema.

La Terapia Cognitiva [1] considera que numerosos problemas se producen por falta de registro de lo que les sucede a las personas.

En el caso de la gestión del tiempo y tareas, la incapacidad de enumerar y registrar los datos referentes a las tareas afrontadas es un síntoma de desorganización. Es por ello que dentro de la terapia cognitiva se recomienda realizar observaciones metódicas y sistemáticas sobre algunos aspectos del problema lo que incrementará el control sobre el propio tiempo y la propia conciencia de lo que se está realizando.

Dentro de la Ingeniería del Software existen técnicas que permiten llevar una mejor organización del trabajo y que pueden ser extensibles a las actividades habituales de cualquier directivo o empleado. Una de las técnicas más destacadas es el Personal Software Process (PSP) [2]. Dentro del PSP se definen un conjunto de técnicas que permiten realizar una mejor planificación y seguimiento del trabajo, estableciendo metas y ofreciendo posibilidad de utilizar un proceso bien definido y medido de trabajo.

Tanto PSP como su extensión a equipos TSP (Team Software Process) [3] están basados en la recopilación de métricas con un elevado nivel de detalle. Una vez que los datos han sido recopilados al nivel necesario, su análisis estadístico permite efectuar una planificación realista, rastreo, predicción, y control de los proyectos y productos de software.

En un contexto similar, se han realizado diferentes experimentos con estudiantes, dentro de alguna asignatura de Ingeniería del Software o afín. Algunos de los más relevantes son los realizados en Méjico [4] y Tailandia [5], útiles para analizar comportamientos en situaciones "ideales", debido a las estrictas condiciones bajo las cuales son realizados. También son destacables otros trabajos como [6] y [7] donde se realizan distintos experimentos controlados con conjuntos de ingenieros de software, analizando el impacto del uso de PSP sobre su habilidad para estimar y planificar su trabajo, la calidad del software que desarrollan y su productividad.
Sin embargo, debido a la complejidad y esfuerzo que la recolección de los datos implica, el Proceso de Software Personal es una técnica que habitualmente, si no se complementa con el uso de herramientas que faciliten su aplicación, puede producir datos de mala calidad, con multitud d[e erratas, quedando demostrado según ciertos estudios [8].

A partir de estos estudios se puede concluir que únicamente el primer nivel de PSP podría llegar a ser usado para mejorar la productividad en un caso real. Por estos motivos, este trabajo se ha centrado en el estudio de los datos brindados por el registro de tiempos especificado en el nivel inferior del PSP. A partir del registros de tiempos se han realizado diferentes experiencias con el fin de mejorar la estimación y la administración del tiempo en el ámbito empresarial, tanto desde el punto de vista del empleado recién contratado, aquellos con varios años de experiencia, y finalmente el directivo encargado de la supervisión del desarrollo de sus correspondientes proyectos.

El resto del trabajo se organiza como sigue. En las siguientes secciones se presentan las experiencias realizadas con empleados en formación, empleados con experiencia y miembros de un equipo de desarrollo para un determinado proyecto, y directivos de proyectos software. Para realizar este estudio se ha contado con los libros de registros de tiempos proporcionados por un conjunto de empleados (con distintos roles) de una empresa real de desarrollo de software, emplazada en Ciudad Real. Finalmente, el trabajo se cerrará con las conclusiones que se pueden extraer del estudio y líneas de trabajo futuro.

\section{ANÁLISIS DE TIEMPOS DE TRABAJO DE EMPLEADOS EN FORMACIÓN}

En el caso de estudio de este trabajo, el autorregistro de actividades utilizado se ha realizado mediante una planilla de tiempos basada en la propuesta del Proceso de Software Personal. En ella se incluye para cada día de trabajo el tiempo efectivo dedicado a cada tarea calculado a partir de la hora de comienzo y de fin, descontándose el tiempo de interrupción.

En la Tabla 1 se observan los campos para el autorregistro utilizado para el empleado en formación. El campo "Día" indica el día de trabajo, "Ini" y "Fin" indican la hora de inicio y de fin. "Int" el 
tiempo gastado en interrupciones. "Tm" indica el tiempo efectivo de trabajo de la sesión (calculado a partir de los datos anteriores). Por último aparecen los datos asociados a cada tarea: "Tec" indica la tecnología asociada para la cual se está realizando la tarea de formación (Java, PHP, .Net, etc.), "Tipo" indica el tipo de tarea realizada (asistencia a curso, realización de ejercicio, reunión para el seguimiento, etc.) y finalmente "Desc" es la descripción propia de la tarea.

Tabla 1. Tabla de autorregistro para empleados en formación.

\begin{tabular}{|l|l|l|l|l|l|l|l|}
\hline Día & Ini & Fin & Int & Tm & Tec & Tipo & Dec \\
\hline & & & & & & & \\
\hline
\end{tabular}

Esta experiencia se ha realizado con empleados programadores en periodo de formación, que está aprendiendo sobre el uso de ciertas tecnologías que le serán necesarias para el desarrollo de su trabajo dentro de la empresa. Dichos trabajadores fueron contratados por una empresa de desarrollo de software de unos 80 empleados, y fueron formados durante un periodo de 3 meses en distintas tecnologías.

Durante este periodo, se les ofreció la posibilidad de realizar parte de dicha formación en su domicilio, mediante el autoestudio de los materiales proporcionados. Además, como introducción se les ofreció un seminario de gestión del tiempo de 2 horas en el que se les explicaron tanto herramientas en las que realizar sus planificaciones semanales de trabajo (por ejemplo, Google Calendar) como el sistema de registro de tiempos planteado.

La actividad consistió en entregar cada lunes durante las tres siguientes semanas un calendario de su planificación semanal de formación para todas las tecnologías requeridas y el registro de tiempos de la semana dedicados a su aprendizaje. Los calendarios de cada semana debían ser entregados el lunes de la semana en cuestión y los registros el lunes siguiente a la conclusión de la semana.

En el seminario se les insistió en la importancia de registrar el tiempo en el que habían interrumpido su formación. De esta manera se ha intentado lograr que fueran conscientes que 1 hora de trabajo no significa 1 hora de formación aprovechada. También se les indicó que había que contar las sesiones de trabajo y no tiempos de desplazamiento o descanso. Se les recomendó a su vez que actualizaran el registro de tiempos de la forma más frecuente posible. Algunos resultados cualitativos relevantes de esta experiencia fueron los siguientes:

- Existía un porcentaje no muy alto de empleados en formación (22\%) que entregaron el mismo plan para todas las semanas y los autorregistros eran muy similares entre sí, conteniendo incongruencias como indicar el estudio de ciertas tecnologías que realmente no le habían sido requeridas. Todos estos datos se descartaron.

- En el análisis de los calendarios se comprobó un cambio radical entre el primer y el segundo calendario semanal. Una posible justificación es el efecto del registro de tiempos tras su primera semana de trabajo.

Algunos resultados cuantitativos se pueden ver en la Tabla 2. Hay que destacar que la actividad se realizó durante las primeras semanas de formación, lo cual explica ciertos resultados.

Tabla 2. Resultados cuantitativos de la experiencia.

\begin{tabular}{|l|c|}
\hline $\begin{array}{l}\text { Media de horas de estudio diario } \\
\text { durante la semana. }\end{array}$ & 8 horas \\
\hline Actividad presencial VS no presencial & $63 \%$ VS 37\% \\
\hline Tiempo medio diario de interrupciones. & 1 hora \\
\hline $\begin{array}{l}\text { Porcentaje de estudio durante el fin } \\
\text { de semana. }\end{array}$ & $3 \%$ \\
\hline
\end{tabular}

El estudio del tiempo dedicado a las tareas autónomas sobre el aprendizaje del lenguaje de programación Java se puede observar en la Figura 1.

\section{Horas estimadas y reales para cada tarea}

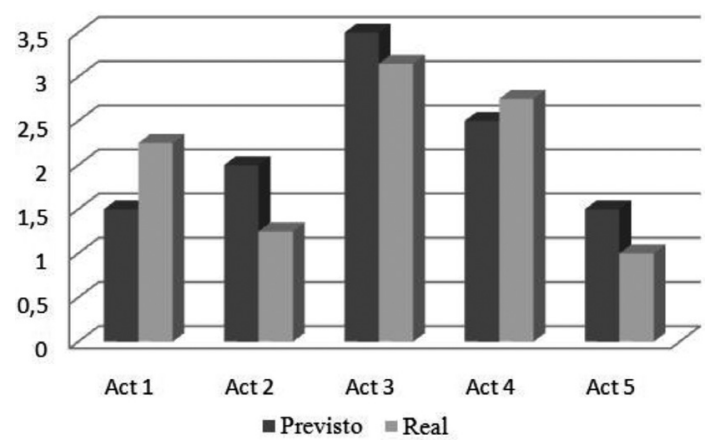

Figura 1. Horas estimadas y reales por cada tarea. 
Como conclusiones se extrajo que la primera tarea, aunque sencilla, costaba mucho a los empleados noveles por ser la primera tarea de formación que realizaban dentro de la empresa.

Lo mismo sucedía con actividades que suponían la introducción a temas tecnológicos complemente distintos a los anteriores, que podían implicar un mayor tiempo de investigación y familiarización (por ejemplo, la actividad 4):

Al final de la actividad los empleados completaron una encuesta anónima sobre la experiencia tras el autorregistro de sus tiempos de trabajo. De entre sus respuestas se pueden destacar los siguientes aspectos:

- Los empleados indicaron que el registro de tiempos les ayudaba a ser conscientes de su uso del tiempo y sobre todo de las interrupciones.

- El tiempo para completar el autorregistro no supone más de 10 minutos si se realiza diariamente. Si se hace al final de la semana es pesado y lento, dando lugar además a un gran número de incorrecciones.

- Como propuesta de mejora recomendaron que fuera completado de forma anónima y que pudieran recibir cierta retroalimentación de su comportamiento.

\section{ANÁLISIS DE TIEMPOS DE TRABAJO DE EMPLEADOS DURANTE DESARROLLO DE UN PROYECTO SOFTWARE}

Para realizar este estudio se ha contado con los datos obtenidos mediante Personal Software Process proporcionados por 5 empleados programadores durante su participación en 5 Proyectos Software. Además, para permitir realizar estudios comparativos y obtener ciertas conclusiones, los 5 proyectos que fueron seleccionados correspondían a proyectos cuyo volumen de trabajo necesario fue estimado en unas 125 horas de desarrollo.

La Tabla 3 representa al registro de tiempos utilizado por cada empleado durante su trabajo en un determinado proyecto de desarrollo de software. Como rasgo distintivo contiene la identificación de la fase del ciclo de vida a la que se asocia la tarea (análisis, diseño, programación, documentación, etc.) y la descripción de la tarea, a partir de la cual es posible hacer su catalogación.
Tabla 3. Tabla de autorregistro para empleados.

\begin{tabular}{|l|l|l|l|l|l|l|l|}
\hline Día & Ini & Fin & Int & Tm & Tec & Tipo & Decs \\
\hline & & & & & & & \\
\hline
\end{tabular}

Tras el estudio detallado de los datos registrados en estos proyectos cabe destacar que el nivel de horas finalmente dedicadas para la realización de cada uno de los proyectos fue realmente entre 200 y 250 horas de trabajo efectivo, muy superior a las 125 horas con las que fueron estimados. Este hecho suele ser habitual, debido al inevitable "factor humano" y el error consecuente en las estimaciones iniciales, así como a la cantidad de imprevistos y dificultades existentes durante el desarrollo del proyecto, no contabilizables en una valoración inicial.

Además, también es posible observar que las características humanas y técnicas de cada empleado son un factor clave para hacer una planificación realista del proyecto. Esto se refleja en los porcentajes dedicados por cada tipo de empleados a las diferentes fases. Estudiando estos porcentajes se pueden distinguir dos tipos de trabajadores, unos que les cuesta tomar contacto con la tecnología (dedican más de un tercio del tiempo en estas fases) y por otro lado los que no tienen problemas con la tecnología pero sí con la realización de documentaciones o, en general, tareas menos técnicas (dedican más de un $35 \%$ a este tipo de tareas).

Una recomendación que se puede extraer del análisis de registros es basar la realización de cualquier proyecto de desarrollo en sesiones de larga duración, lo cual no es siempre fácil.

En los casos estudiados las sesiones tienen un promedio de 3 horas 15 minutos de tiempo efectivo, lo cual ha hecho que en más de un 30\% de sesiones se haya logrado terminar las tareas.

Por el contrario, la gran duración de las sesiones las hace más propensas a las interrupciones. De esta manera, se observa que el porcentaje de sesiones con interrupción ha sido de alrededor del $80 \%$ el con una media de 14 minutos de interrupción por sesión.

También se puede comprobar que aparecen más errores en las tareas que se realizaron con un mayor número de interrupciones. 
En cuanto a la distribución semanal del trabajo los empleados que no asisten a cursos de formación o participan en otros trabajos, realizan sus tareas durante los días de la semana, siempre en días como el lunes y el jueves y solo puntualmente extienden su jornada laboral al fin de semana para la conclusión de alguna actividad muy puntual.

Por el contrario los que asisten a cursos de formación propuestos por la empresa, o realizan otro tipo de actividad adicional, frecuentemente se ven obligados a continuar sus actividades profesionales del ámbito empresarial fuera de su horario laboral incluyendo fines de semana.

Todos estos aspectos son imprescindibles para realizar planificaciones realistas previas al inicio y a la realización de proyectos software.

\section{ANÁLISIS DE TIEMPOS DE TRABAJO DE UN DIRECTIVO DE PROYECTOS DE DESARROLLO SOFTWARE}

La experiencia realizada con un directivo y mánager de proyectos de desarrollo de software se ha extendido durante más tiempo que las realizadas sobre los cinco empleados analizados con rol de programador. En este caso se cuenta con registros de tiempos desde Enero hasta Septiembre de 2015, incluyendo la siguiente distribución relativa al número de proyectos dirigidos y el número de empleados programadores involucrados en ellos.

Tabla 4. Proyectos y trabajadores gestionados durante el estudio.

\begin{tabular}{|c|c|c|}
\hline Periodo & $\begin{array}{c}\mathbf{N}^{\circ} \text { de } \\
\text { Proyectos }\end{array}$ & $\begin{array}{c}\mathbf{N}^{\circ} \text { de } \\
\text { Trabajadores }\end{array}$ \\
\hline $01 / 01 / 2015-31 / 03 / 2015$ & 5 & 16 \\
\hline $01 / 04 / 2015-30 / 06 / 2015$ & 6 & 21 \\
\hline $01 / 07 / 2015-30 / 09 / 2015$ & 3 & 15 \\
\hline
\end{tabular}

El registro utilizado por el directivo tiene como singularidad una descripción de la tarea utilizando dos campos "Tipo" (Gestión, Formación, Reunión, Investigación) y Descripción.

Tabla 5. Tabla de autorregistro para el directivo del proyecto.

\begin{tabular}{|l|l|l|l|l|l|l|l|}
\hline Día & Ini & Fin & Int & Tm & Tec & Tipo & Decs \\
\hline & & & & & & & \\
\hline
\end{tabular}

Los resultados de agregar los datos de estos registros se pueden observar en la Figura 2.

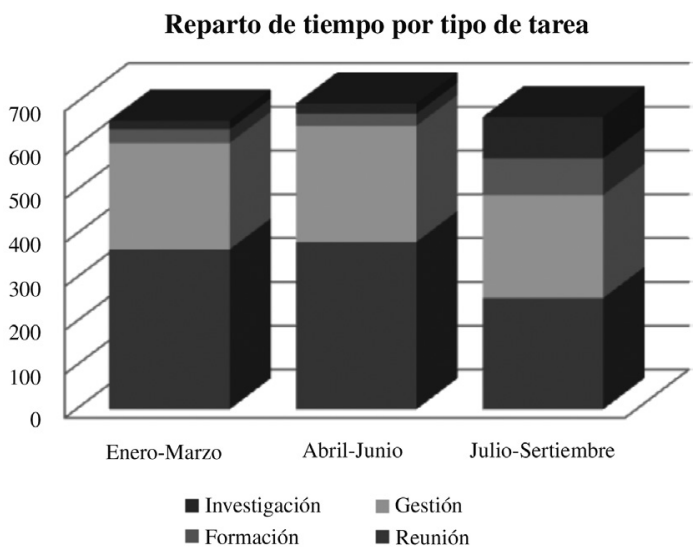

Figura 2. Horas estimadas y reales para cada tarea.

El tiempo de trabajo total en los diferentes cuatrimestres se mantiene prácticamente constante. El tiempo de trabajo semanal para cada uno de los trimestres es de 44 horas, superior a las 40 horas de una jornada laboral estipulada, teniendo en cuenta además, que se trata de horas de trabajo real, por lo que hay que tener en cuenta que en la mayoría de trabajos para conseguir 40 horas de trabajo real, descontando interrupciones y descansos se necesitarían jornadas laborales de entre 45 y 50 horas.

A su vez, observando la distribución de tareas por tipo, se ha comprobado como la preparación de reuniones y demás tareas de gestión, requieren casi la totalidad del tiempo de trabajo dedicado por el directivo. Por el contrario, tareas referentes a su propia formación mediante cursos y seminarios, o posibles tareas de investigación de modo autónomo, habitualmente no suponen más de un $15 \%$ de su tiempo de trabajo.

Además, analizando los datos más en detalle se pueden extraer conclusiones bastante útiles para la organización del trabajo del mánager.

Por ejemplo, se puede identificar la influencia del tiempo dedicado a Reuniones con el resto de trabajadores (en este caso bastante alto, entorno al $50 \%$ ) y como se reparte este tiempo entre reuniones presenciales y no presenciales mediante herramientas basadas en el uso de internet. En 
este caso se divide por igual entre presenciales y no presenciales.

Además, se puede identificar el incremento del tiempo dedicado a Reuniones durante los meses en los que el directivo tiene que gestionar un mayor número de proyectos y personas (primer y tercer trimestre). Tener en cuenta esta relación puede resultar importante, ya que la dedicación de modo mayoritaria a la preparación de Reuniones podría tener consecuencias problemáticas para el resto de actividades (Investigación, Formación y Gestión), ya que el tiempo dedicado a ellas se vería necesariamente reducido.

Otro de los puntos importantes es establecer objetivamente cuáles son los picos de trabajo del directivo con el fin de que este pueda organizar el resto de sus tareas para poder afrontarlos. En este caso se han identificado las segundas quincenas de Marzo y Junio como puntos de máxima dedicación a tareas de Gestión, coincidiendo con el periodo en el que el número de proyectos dirigidos fue mayor.

Por último, se ha estudiado la influencia de festivos o viajes imprevistos, comprobándose cómo semanas con más de un $25 \%$ de días con festivos o con desplazamientos resultaban ser cerca de un $33 \%$ menos productivas.

\section{CONCLUSIONES}

En este trabajo se han presentado ciertas experiencias en la utilización del registro de tiempos especificado en el Proceso de Software Personal. Concretamente se han estudiado casos tanto de empleados sin y con experiencia como del propio directivo o mánager responsable del seguimiento y gestión de proyectos. Para ello se han analizado cuadernos de registros de tiempos proporcionados por este tipo de profesionales pertenecientes a una empresa real de desarrollo.

Tras este estudio, se han obtenido un conjunto de conclusiones, principalmente relativas a cómo periodo de alto estrés afectan a la productividad en próximas semanas. Asimismo, se han obtenido un conjunto de recomendaciones relativas al modo de trabajo y gestión de tiempo efectuado por cada tipo de trabajador ante distintas situaciones y periodos de tiempo, que podrían llegar a ayudar a mejorar su propia gestión.

Como trabajo futuro se plantea la construcción de una aplicación web que sirva como observatorio del tiempo donde los empleados puedan registrar los tiempos trabajados con respecto a sus tareas y puedan recibir una retroalimentación del sistema obtenida a través del análisis inteligente y automático de los datos que facilitan. De este modo, además de facilitar el seguimiento y uso de PSP en su primer nivel, se conseguiría reducir la tasa de errores en el registro de tiempos, mejorando su calidad, y pudiéndose ser usados con unas mejores garantías para su posterior proceso de análisis y de extracción de conocimiento.

\section{REFERENCIAS}

[1] J. Beck y A. T. Beck. "Terapia cognitiva: conceptos básicos y profundación". GEDISA. Barcelona, España, pp. 389. 2009. ISBN: 968852123X.

[2] W. Humphrey. "PSP, A Self-Improvement Process for Software Engineers". AddisonWesley. 2005. ISBN: 0321305493.

[3] W. Humphrey. "TSP, Leading a Development Team". Addison Wesley. 2006. ISBN: 0321349628.

[4] O. Gómez, A. Aguileta, G. Gómez y R. Aguilar. "Estudio del Proceso Software Personal (PSP) en un entorno académico". Revista Electrónica de Computación, Informática, Biomédica y Electrónica (ReCIBE). Vol. 2, pp. 324-236. Mayo 2014. ISSN: 2007-5448.

[5] S. Ramingwong and K. Ramingwong. "Implementing a Personal Software Process (PSPSM) Course: A Case Study". Journal of Software Engineering and Applications. Scientific Research Publishing. Vol. 5, pp. 639-644. Agosto 2012. ISSN: 1947-3931. DOI: $10.4236 /$ jsea.2012.58074.

[6] F. Grazioli, D. Vallespir, L. Pérez and S. Moreno. "The Impact of the PSP on Software Quality: Eliminating the Learning Effect Threat through a Controlled Experiment". Advances in Software Engineering. Elsevier. Vol. 2014. pp. 156166. September, 2014. ISSN: 0965-9978. DOI: $10.1155 / 2014 / 861489$. 
[7] D. Vallespir, F. Grazioli, L. Pérez and S. Moreno. "Demonstrating the impact of the PSP on software quality and effort: eliminating the programming learning". TSP Symposium, Software Engineering Institute, Carnegie Mellon University USA. 2013.
[8] C. Valverde, F. Grazioli y D. Vallespir. "Un estudio de la calidad de los datos recolectados durante el uso del personal software process". Proceedings of the 9th Jornadas Iberoamericanas de Ingeniería de Software e Ingeniería del Conocimiento. Uruguay. 2012. 